\title{
Characterization of porous media based on the polarimetric matrix models
}

\author{
Sergey Savenkov ${ }^{1}$, Alexander Priezzhev ${ }^{2}$, Yevgen Oberemok $^{1}$, Ivan Kolomiets ${ }^{1}$, Pertti Silfsten ${ }^{3}$, \\ Tuomas Ervasti ${ }^{4}$, Jarkko Ketolainen ${ }^{4}$, and Kai-Erik Peiponen ${ }^{3}$ \\ ${ }^{1}$ Faculty of Radio Physics, Electronics and Computer Systems, Taras Shevchenko National University of Kyiv, 64 \\ Vladimirskaya str., Kiev 01033, Ukraine \\ 2 Department of Physics and International Laser Center, Lomonosov Moscow State University, Vorobyovy Gory, \\ Moscow 119992, Russia \\ ${ }^{3}$ Department of Physics and Mathematics, University of Eastern Finland, PO Box 111, FI-80101 Joensuu, Finland \\ ${ }^{4}$ School of Pharmacy, Promis Centre, University of Eastern Finland, PO Box 1627, FI-70211 Kuopio, Finland \\ *e-mail: sns@univ.kiev.ua
}

\begin{abstract}
In this paper, we measured the Mueller matrices of the samples with different porosity in the visible range (the wavelength of the input radiation $632.8 \mathrm{~nm}$ ) for the observation angles from $5^{0}$ to $85^{\circ}$. The samples under study were the tablets made of microcrystalline cellulose with the known values of porosity. To characterize the depolarizing properties of the studied samples we used the matrix models of depolarizing medium, in which the Mueller matrices describing depolarization have different structure and include different numbers of parameters. It is shown that Mueller polarimetry ensures at least $5 \%$ accuracy of porosity identification for this class of objects at the wavelength $632.8 \mathrm{~nm}$. (C) 2017 Journal of Biomedical Photonics \& Engineering.

Keywords: Mueller matrix; porosity; depolarization; linear birefringence; circular birefringence.

Paper \#3165 received 28 Feb 2017; revised manuscript received 12 Apr 2017; accepted for publication 13 Apr 2017; published online 28 Apr 2017. doi: 10.18287/JBPE17.03.010306. [Special Issue. Years in Biophotonics: 70th Anniversary of Prof. A.V. Priezzhev].
\end{abstract}

\section{References}

1. L. V. Wang, G. L. Cote, and S. L. Jacques, "Special Section Guest Editorial," J. Biomed. Opt. 7(3), 278 (2002).

2. V. V. Tuchin, L. V. Wang, and D. A. Zimnyakov, Optical Polarisation in Biomedical Applications, Springer, Berlin (2006).

3. N. Ghosh, and I. A. Vitkin, "Tissue polarimetry: concepts, challenges, applications, and outlook," J. Biomed. Opt. 16(11), 110801 (2011).

4. S. N. Savenkov, "Mueller Matrix Polarimetry in Material Science, Biomedical, and Environmental Applications," Handbook of Coherent-Domain Optical Methods, V. V. Tuchin (ed.), Springer, New York, 1175-1253 (2013).

5. J. J. Gil, and E. Bernabeu, “A depolarisation criterion in Mueller matrices," Opt. Acta 32(3), 259-261 (1985).

6. R. Espinosa-Luna, and E. Bernabeu, "On the Q(M) depolarisation metric," Opt. Commun. 277(2), 256-258 (2007).

7. S. R. Cloude, and E. Pottier, "Concept of polarisation entropy in optical scattering," Opt. Eng. 34(6), 15991610 (1995).

8. R. Ossikovski, “Alternative depolarisation criteria for Mueller matrices,” J. Opt. Soc. Am. A 27(4), 808-814 (2010).

9. R. A. Chipman, "Depolarization index and the average degree of polarization," Appl. Opt. 44(13), 2490-2495 (2005). 
10. M. F. G. Wood, N. Ghosh, E. H. Moriyama, B. C. Wilson, and I. A. Vitkin, "Proof-of-principle demonstration of a Mueller matrix decomposition method for polarised light tissue characterization in vivo," J. Biomed. Opt. 14(1), 014029 (2009).

11. N. Ghosh, M. F. G. Wood, S.-H. Li, R. D. Weisel, B. C. Wilson, R.-K. Li, and I. A. Vitkin, "Mueller matrix decomposition for polarised light assessment of biological tissues," J. Biophoton. 2(3), 145-146 (2009).

12. N. Ghosh, M. F. G. Wood, and I. A. Vitkin, "Mueller matrix decomposition for extraction of individual polarisation parameters from complex turbid media exhibiting multiple scattering, optical activity, and linear birefringence," J. Biomed. Opt. 13(4), 044036 (2008).

13. S. Y. Lu, and R. A. Chipman, "Interpretation of Mueller matrices based on polar decomposition," J. Opt. Soc. Am. A 13(5), 1106-1113 (1996).

14. P. Silfsten, V. Kontturi, T. Ervasti, J. Ketolainen, and K.-E. Peiponen, "Kramers-Kronig analysis on the real refractive index of porous media in the terahertz spectral range," Opt. Lett. 36(5), 778-780 (2011).

15. C. F. Bohren, and E. R. Huffman, Absorption and scattering of light by small particles, Wiley, New York (1983).

16. M. I. Mishchenko, J. W. Hovenier, and L. D. Travis, Light scattering by nonspherical particles, Academic Press, San Diego (2000).

17. A. A. Kokhanovsky, Light Scattering Media Optics: Problems and Solutions, Praxis Publishing, Chichester (2001).

18. M. I. Mishchenko, and L. D. Travis, "Polarisation and depolarisation of light," in Light Scattering from Microstructures, F. Moreno, and F. González (eds.), Springer-Verlag, Berlin, 159-175 (2000).

19. K. Kim, L. Mandel, and E. Wolf, "Relationship between Jones and Mueller matrices for random media," J. Opt. Soc. Am. A 4(3), 433-437 (1987).

20. S. N. Savenkov, "Mueller-matrix characterization of biological tissues," in Polarimetric Detection, Characterization and Remote Sensing NATO Science for Peace and Security Series C: Environmental Security, Springer, Netherlands, 437-472 (2011).

21. M. Williams, "Depolarisation and cross polarisation in ellipsometry of rough surfaces," Appl. Opt. 25(20), 3616-3622 (1986).

22. R. Ossikovski, "Analysis of depolarising Mueller matrices through a symmetric decomposition,” J. Opt. Soc. Am. A. 26(5), 1109-1118 (2009).

23. S. N. Savenkov, "Optimization and structuring of the instrument matrix for polarimetric measurements," Optical Engineering 41(5), 965-972 (2002).

\section{Introduction}

The results recently obtained in polarimetry clearly demonstrate the availability of polarimetric methods for studying the properties of biological objects [1-3]. It is known that biological objects are characterized by complex "polarization behavior" [2]. In particular, the objects of this class can demonstrate both linear and circular birefringence and depolarization. The interaction of polarized electromagnetic radiation with biological objects is characterized by high depolarization, and, more importantly, depends on the input polarization state.

The dependence of depolarization of the output radiation on the input polarization state is referred to as anisotropic depolarization. The most common method to characterize the depolarizing properties of objects is to use the so-called single value depolarization metrics [4]. All existing depolarization metrics can be divided into two classes. The first class is the metrics including only the elements of the Mueller matrix of the studied object, e.g., the depolarization metric [5], the $Q$-metric [6], the Cloude [7] and Lorentz [8] entropy. The second class is the metrics involving the scanning of all possible states of input polarizations, see, e.g., Ref. [9].
It should be borne in mind that the single value depolarization metrics carry some averaged information on the depolarization. This means that in case of anisotropic depolarization the information capabilities of single value depolarization metrics appear to be limited. Moreover, in the literature the cases are described, when certain metrics generally do not adequately characterize the depolarizing properties of the studied object [8].

Therefore, to characterize the polarization properties of biological objects and, in particular, their depolarization capabilities, the matrix models of depolarizing medium show considerable promise.

In this case, the Mueller matrix of the object is represented as a matrix product of, using the terminology Jones [4], elementary types of anisotropy, i.e., the linear and circular birefringence and dichroism, and the matrices describing depolarization. In Refs. [1012] the authors demonstrated the possibility of a matrix model based on the so-called generalized polar decomposition theorem [13] to obtain information on linear and circular birefringence in high depolarization of the output radiation.

In this paper, we study the sensitivity of the Mueller method and various depolarization matrices to the porosity of samples, which are tablets made of a 
common pharmacological filler, the microcrystalline cellulose [14]. The paper is organized as follows. Section 2 outlines the existing Mueller matrix models of depolarizing objects. In Section 3 the detailed information about the porous samples under consideration is presented. Section 4 presents the results of measurements, comparative analysis and discussion.

\section{Matrix models of depolarizing objects}

The propagation of electromagnetic radiation in an inhomogeneous medium, alongside with the change in the polarization state, is also accompanied by the "removal" of a portion of the input radiation energy, which is spatially redistributed over the different directions of propagation, i.e., the radiation is scattered [15-17]. The scattering is usually accompanied by the depolarization of the output radiation $[15,18]$. The depolarizing capability is a property of the studied object, which manifests itself as a decrease of the polarization degree of the input completely polarized radiation after its interaction with the object.

There are two types of matrix models of depolarizing objects, the additive and the multiplicative ones. The additive matrix models are associated with the discrete approximation of the medium, i.e., the studied medium is represented as a set of scatterers. These models are based on the weighted summation of deterministic Mueller matrices describing the interaction of radiation with individual scatterers [19, 7]. In this paper, this type of model is not considered.

The multiplicative models represent the studied medium (deterministic andlor depolarizing) as a structure consisting of a number of layers, successive with respect to the propagation direction of the input radiation. Each layer is described by the Mueller matrix of only one type of anisotropy and lor depolarization [20].

Apparently, the first multiplicative matrix model of depolarizing medium was the model proposed in Ref [21]. In this model the Mueller matrix of depolarizing medium is presented as a product of deterministic Muller matrices, describing linear birefringence, linear dichroism, etc., and the depolarization matrix of the form

$$
\left(\begin{array}{cccc}
1 & A_{1} & A_{2} & A_{3} \\
0 & P_{1} & 0 & 0 \\
0 & 0 & P_{2} & 0 \\
0 & 0 & 0 & P_{3}
\end{array}\right)
$$

The diagonal elements of the matrix (1) represent the degree of the following input polarizations: horizontal and vertical, linear with the azimuths $\pm 45^{\circ}$, and the left and right circular ones. The quantities $A_{1}, A_{2}$, and $A_{3}$ in the first row of the matrix (1) are quantitative estimates of depolarization asymmetry for the above three pairs of orthogonal input polarizations.
The model most widely used to date is the multiplicative model of depolarizing medium proposed in Ref. [13]. In this model $\mathbf{M}=\mathbf{M}_{\Delta} \mathbf{M}_{R} \mathbf{M}_{D}$, where $\mathbf{M}_{R}$ is the matrix of a retarder, $\mathbf{M}_{D}$ is the matrix of a diattenuator, and the matrix:

$$
\mathbf{M}_{\Delta}=\left(\begin{array}{cc}
1 & \overrightarrow{\mathbf{O}}^{T} \\
\overrightarrow{\mathbf{P}}_{\Delta} & \mathbf{m}_{\Delta}
\end{array}\right), \quad \mathbf{m}_{\Delta}^{T}=\mathbf{m}_{\Delta},
$$

describes the depolarizing properties of the studied object. This model is referred to as the generalized polar decomposition. In Eq.(2) the vector $\overrightarrow{\mathbf{P}}_{\Delta}$ describes the polarization state "generated" by the Mueller matrix (2), when the input radiation is unpolarized.

In Ref. [23] the model of depolarizing medium, described by the symmetrical expansion of its Mueller matrix, is proposed:

$$
\mathbf{M}=\mathbf{M}_{D 2} \mathbf{M}_{R 2} \mathbf{M}^{D A} \mathbf{M}_{R 1}^{T} \mathbf{M}_{D 1},
$$

where the symbol " $T$ " denotes the transposition of the matrix $\mathbf{M}_{R 1} ; \mathbf{M}_{D 1}, \mathbf{M}_{D 2}$ and $\mathbf{M}_{R 1}, \mathbf{M}_{R 2}$ are the matrices of two elliptical polarizers and phase plates, respectively; the matrix $\mathbf{M}^{D A}$ is a depolarization matrix having the form

$$
\mathbf{M}^{D A}=\left(\begin{array}{cccc}
m_{11} & 0 & 0 & 0 \\
0 & m_{22} & 0 & 0 \\
0 & 0 & m_{33} & 0 \\
0 & 0 & 0 & m_{44}
\end{array}\right) .
$$

One can see that the depolarization matrices (1), (2), and (4) have different structures and contain a different number of parameters. The analysis aimed at the interpretation of the physical properties of depolarizing objects based on the above matrix models [13, 21, 22] is beyond the scope of this paper. Below we use the depolarization matrices (1), (2), and (4) to assess their sensitivity to the porosity degree of the considered samples.

\section{Objects of study and experimental technique}

In this paper, we investigate the porous samples made in the form of tablets [14]. The tablets (the diameter $13 \mathrm{~mm}$, the thickness $2 \mathrm{~mm}$, and the porosity $40.1 \%$; $35.1 \% ; 30.7 \% ; 26.7 \%$, and $23.2 \%$ ) were made using the press PUUMAN PCS-1 from microcrystalline cellulose (Avicell PH-200 FMC Biopolymer), a filler commonly used in pharmaceutical industry to produce tablets. 


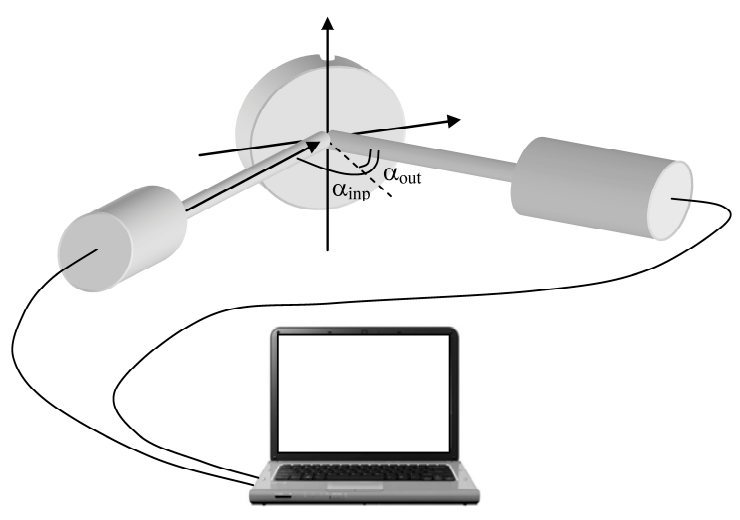

(a)

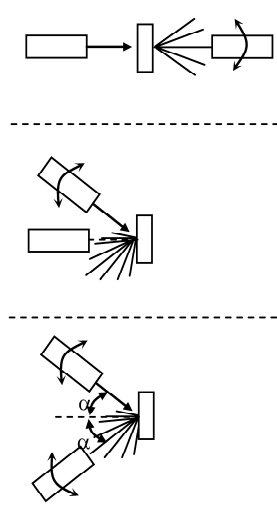

(b)

Fig. 1 (a) Schematic diagram of the Mueller polarimeter; (b) geometry of experiment.

The compression was implemented using the cylindrical punches. The variation of the sample porosity was achieved by changing the cellulose powder mass in the range from $397 \mathrm{mg}$ to $512 \mathrm{mg}$ with simultaneous change of the upper punch contour, providing the stability of the sample thickness. The compression speed was $100 \mathrm{~mm} / \mathrm{s}$. After one day of compression, the dimensions and weight of the tablets were measured, making it possible to calculate the density of each sample. The absolute density of samples was determined using the pycnometer Quantachrome MVP-1.

The schematic diagram of the Mueller polarimeter is shown in Fig. 1a.

The polarized electromagnetic radiation was generated by the He-Ne laser $(\lambda=632.8 \mathrm{~nm})$. The input channel was composed of a fixed prism polarizer and two phase plates, implemented as controlled liquid crystal cells with different azimuths of orientation and values of the phase shift.

The receiving channel consisted of the continuously rotating crystal phase plate, the fixed prism polarizer orthogonal to the one in the input channel, and a photodetector. Thus, the receiving channel was actually a full-Stokes polarimeter [23].

During the measurements, various options of the experimental geometry were tested (see Fig. 1b). The geometry with similar input and observation angles (the lower part of Fig.1b) appeared to be the most informative. For the geometry shown in the top part of Fig. $1 \mathrm{~b}$, the measurement at the wavelength $\lambda=632.8 \mathrm{~nm}$ and the sample thicknesses of $2 \mathrm{~mm}$ are also possible. However, in this case the output radiation is completely depolarized for all the states of the input polarization.

For all five samples we have measured the complete Mueller matrices in the range of observation and input angles from $5^{\circ}$ (this value was determined by the design constraints of the polarimeter receiving channel) to $85^{\circ}$ (for the observation angles $85^{\circ}-90^{\circ}$ the intensity of scattered radiation is vanishingly small).

Before the measurement in each sample, the calibration measurements of the Mueller matrix were carried out in test objects, i.e., the empty space, the prism polarizer, etc. The Mueller matrices for each of the observation angles, analyzed in the next section, were obtained as a result of averaging over five hundred measurements. The measurement error of the matrix elements did not exceed $1 \%$.

\section{Results and Discussion.}

Based on the measured Mueller matrices, the depolarization matrices (1), (2) and (4) were calculated for all observation angles. Figures $2 \mathrm{a}, \mathrm{b}$, and c present the non-zero elements versus the observation angle for the matrices (1), (2) and (4), respectively.

As seen from Fig. 2, at small observation angles, $5^{\circ}-25^{\circ}$, the situation is close to the isotropic depolarization, which is to the isotropic factor described by the following Mueller matrix

$$
\mathbf{M}=\left(\begin{array}{cccc}
1 & 0 & 0 & 0 \\
0 & 0.2 & 0 & 0 \\
0 & 0 & 0.2 & 0 \\
0 & 0 & 0 & 0.2
\end{array}\right) .
$$

The possible explanation is that the multiple volume scattering dominates in this case and the degree of polarization of the output radiation is only slightly dependent on the input polarization state.

At large observation angles, $70^{\circ}-85^{\circ}$, all depolarization matrices (1), (2), and (4) degenerate into the unit matrix. The reason is that in this case the single scattering from the surface of the tablets is dominant, and the depolarization of the scattered radiation is minimal or absent. 

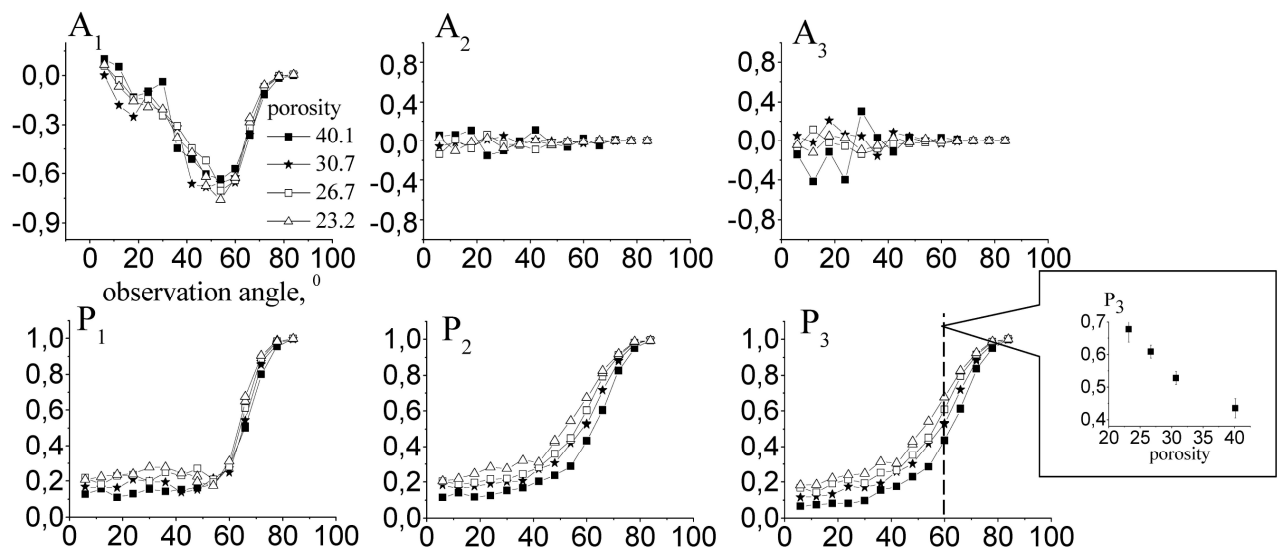

(a)
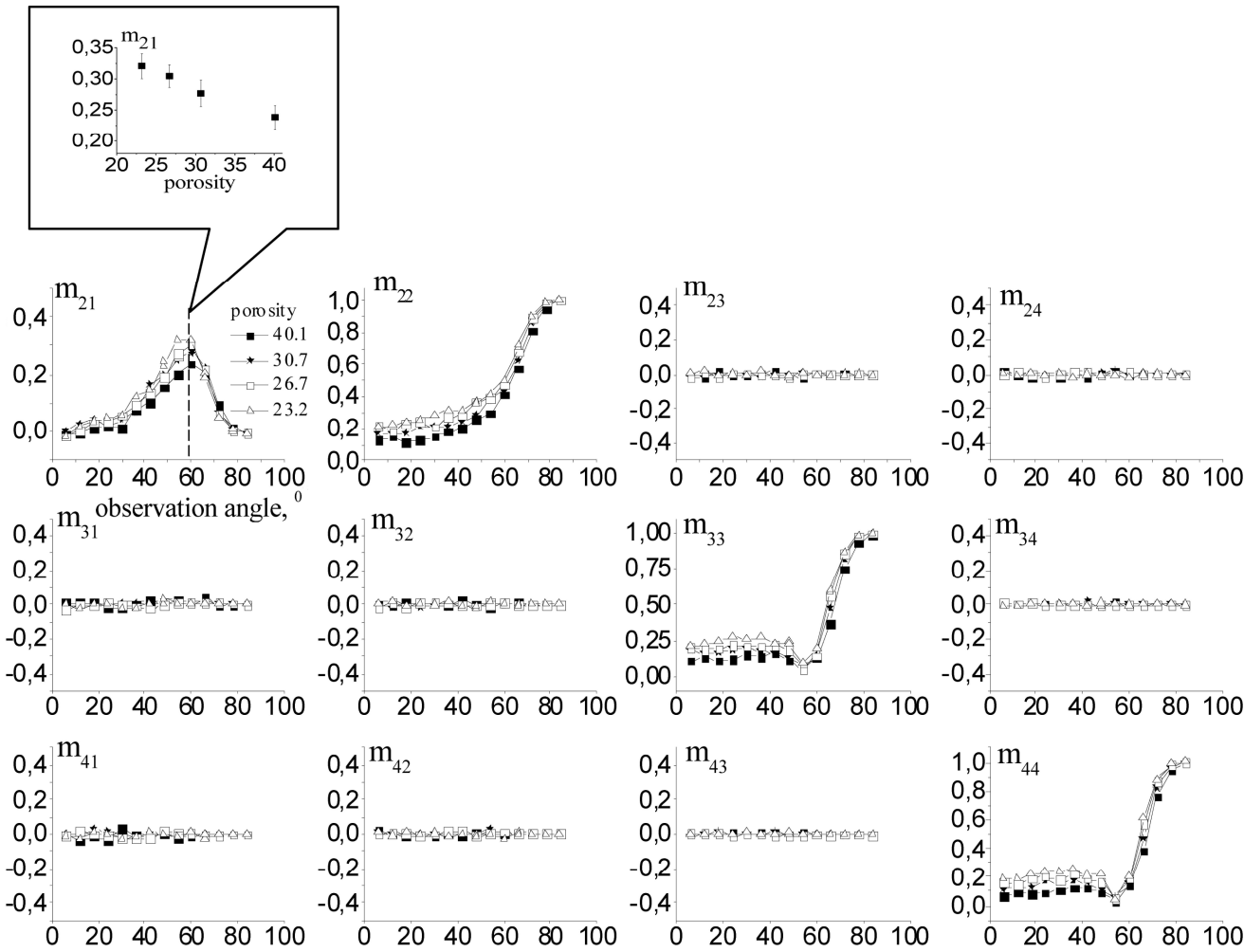

(b)
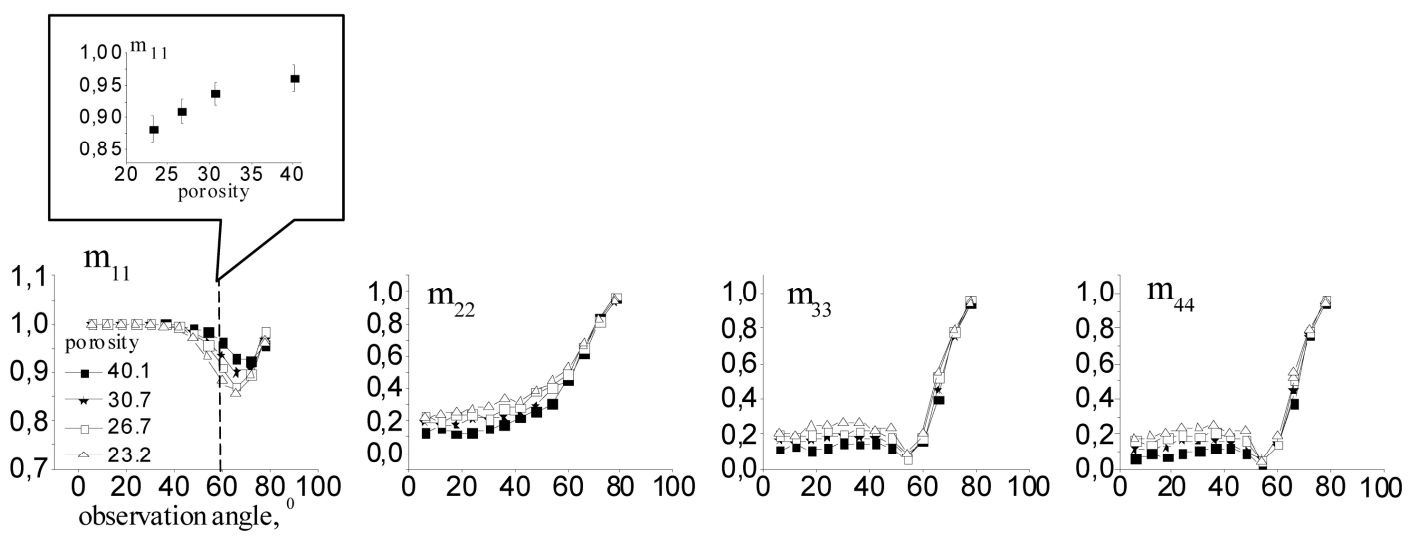

(c)

Fig. 2 Dependence of nonzero elements of the matrices (1), (2), and (4) on the observation angle. 


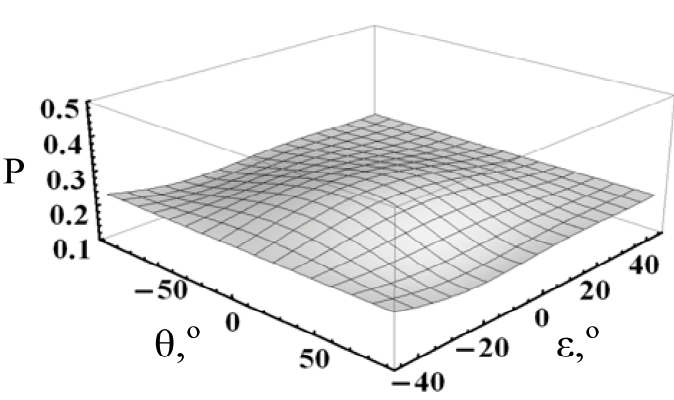

(a)

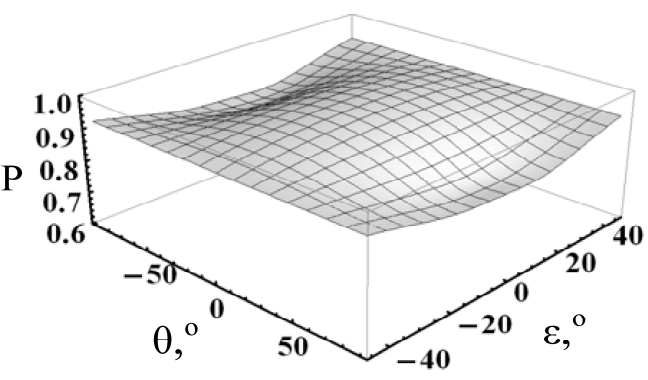

(b)

Fig. 3 Dependence of the output degree of polarization on the azimuth $\theta$ and the ellipticity $\varepsilon$ of the input polarizations for the sample with the porosity of $23.2 \%$ and the observation angles $18^{\circ}$ (a) and $72^{\circ}$ (b).

Note that for the depolarization matrices (1) and (2), having no nonzero off-diagonal elements, only the elements $m_{12}$ in the matrix (1) and $m_{21}$ in the matrix (2) vary depending on the observation angle. For the diagonal elements $m_{22}, m_{33}$, and $m_{44}$ the dependence on the observation angle is quite similar and determined by the interrelation between the volume and the surface scattering for a given observation angle.

In our opinion, this results from the fact that the depolarization, by which the samples under consideration are characterized, is weakly dependent on the input polarization. In other words, in this case, the depolarization is weakly anisotropic. This fact is illustrated by the dependences of the output degree of polarization on the azimuth and elasticity of the input polarizations, presented in Fig. 3.

For the depolarization matrix (2), if the input electromagnetic radiation is unpolarized, then the output radiation is partially polarized linearly and horizontally. The maximal value of the polarization degree varies from $25 \%$ to $35 \%$, depending on the porosity, and is achieved within the range of observation angles from $50^{\circ}$ to $60^{\circ}$.

In each of the cases, illustrated by Figs. 2 and 3, there are matrix elements and ranges of observation angles that allow reliable identification of samples having different porosity. However, the sensitivity to the sample porosity is rather different in the considered models (see the corresponding insets in Figs. 2 and 3).

\section{Conclusion.}

The obtained results demonstrate that the Mueller polarimetry ensures the identification of the porosity for a given class of objects at the wavelength $\lambda=632.8 \mathrm{~nm}$ of the input radiation with the accuracy better than $5 \%$. The next step would be to conduct similar studies for other wavelengths of the input electromagnetic radiation. 